\title{
The Distribution Character of No.12 Coal Mine and Gas in Pingdingshan
}

\author{
Wei Wang ${ }^{1,2}$, Jiang-wei Yan $^{1,2}$ \\ ${ }^{1}$ State Key Laboratory Cultivation Base for Gas Geology and Gas Control \\ (Henan Polytechnic University), \\ Jiaozuo 454003, China \\ ${ }^{2}$ Institute of Gas-geology, \\ Henan Polytechnic University, \\ Jiaozuo 454003, China
}

\begin{abstract}
The extruding of coal and gas is related to the geologic structure, which controls the storage of gas and shows the damage extent of coal seam and width distribution, as well as the distribution of coal and gas extruding. Based on lots of information about gas area and gas parameters, starting from the essential character of Pingdingshan mine area, like the No.10 mine sycline, Guozhuang anticline, Likou sycline, this article analyses the main controlling role of structure on the No.12 coal mine and gas, finding the distribution regularities of the No.12 coal mine and gas, and made some speculation about potential danger in the virgin area.
\end{abstract}

Keywords-the outburst of coal and gas; controlling role; distribution character; fold structure; the storage of gas

China has the most serious coal and gas accidents in the world; the death rate of coal mine accidents remains the first place ${ }^{[1]}$. According to the Statistics of 1:2.5 Million Coal Mine and Gas Structure, our outburst of mine field increased from 274 pairs of 1990 to 1146 pairs of 2011, averagely increasing by 43 pairs every year. Outburst of coal and gas happened almost 280 times every year ${ }^{[1]}$, which makes it the first disaster of gas disaster. ${ }^{[2]}$

Outburst of coal and gas is a kind of extremely complicated phenomenon of losing stability happens underground. Nowadays, there are still doubts about principle of outburst around the experts in the world, putting forward all kinds of assumption like the stress role theory, the gas role theory and the comprehensive role theory. Among these, the comprehensive role theory is acknowledged by most scholars. It talks that the gravity, gas and coal mine make the outburst. The stress is the leading factor causing the outburst. High pressured gas plays a decisive role in the development. The physical substance of coal is the factor that stops the outburst. ${ }^{[3-9]}$

Pingdingshan mine area is one of the very complicated areas in China. Pingdingshan the No.12 mine is the most serious outburst coal areas. Many experts did research into Pingdingshan mine area; this article uses the gas ground theory and coal dynamics way to analyze and makes area prediction about the potential danger on the virgin area.

\section{Pingdingshan Mine Area Summary}

Controlled by the area structure, Pingdingshan mine area's main character is top in the middle and deep around. The mine area is formed into a series of "north west west-north west” folds (Lushan anticline, Likou syncline, Xiangjia anticline, Jing Jiawa syncline) ,arranged in parallel, with the "north west west-north west" breaks (Luye break, Xiangjia break, Shangshui breakage, Zhangde breakage) and "North north east-North east break" (Jiaxian break, Luogang break). The structure of NWW-NW is shown as the squeeze of north south direction and large scales of thrust nappe. The structure of NNE, NE is early shown as compression twist. In comparison, the constructure of NNW-NW lasts long and appears heavily and spreads the whole area. The distribution of mine area structure and mine field is shown in Figure 1.

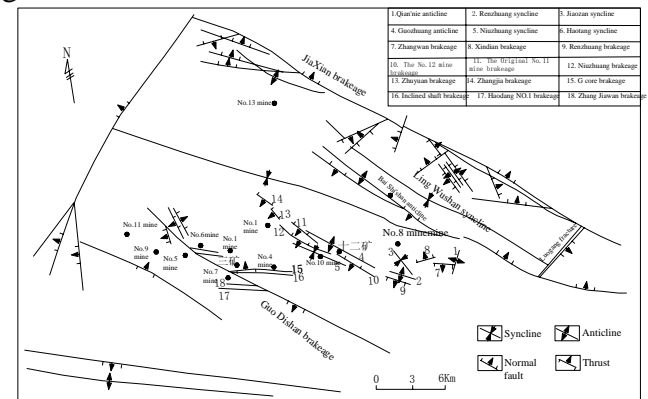

Figure 1. Structure and mine distribution map of Pingdingshan mining area

\section{Mine Field GeOlogic Structure AND DISTRIBUTION CHARACTERISTICS}

The No.12 mine field is located at the east of Pingdingshan mining area, the No.8 mine field is on the east and the No.10 mine field is on the west. There are two sunsidiary folds in the mine field, such as Niuzhuang syncline and Guozhuang syncline. Three large and medium-size reverse faults as Niuzhuang reverse fault, F2 reverse fault, the original No.11 reverse fault are compression and twist structure in the direction of NW-SE. The whole mine field is ran through by Niuzhuang syncline and Guozhuang syncline which are 
compression and twist structure in the direction of NW-SE and control the first and second level of the mine field; the third level is located at the transition area of Likou syncline east end of convergence, which is belong to stress concentration belt and the medium and minor fault is controlled by it. The No.12 mine field is belonging to the complex structure area of Pingdindshan mining area, and the structure and distribution are showing in Figure 2.

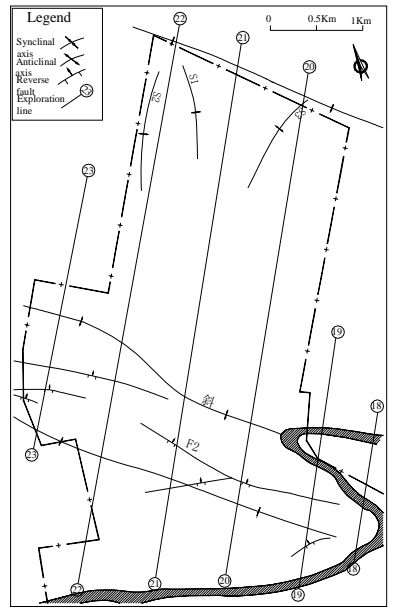

Figure 2. Structure outline plans of the No.12 mine of Pingdingshan

\section{The No.12 Mine AND Gas OutBurst Distribution} RULE

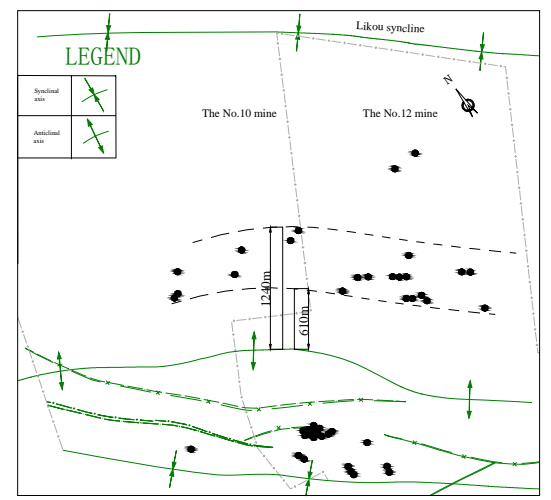

Figure 3. The No.12 mine geologic structure outline and outburst distribution diagram

The Figure 3 is the No.12 mine geologic structure and outburst distribution diagram and the content is boundary of group F; the No.10 mine group F coal seam is located at the west of the No.12 mine group F. The No.10 mine extruded for 50 times and the number of outburst accounts for $12 \%$, the number of pulsion accounts for $86 \%$ and the number of dump accounts for $2 \%$. Group D and group E are all small outburst and almost for pulsion; group $\mathrm{F}$ is medium outburst or outsize outburst. There are 27 times coal and gas outburst and 9 times of dynamic phenomenon in No.12 mine.

The author puts the outburst points of three main coal mining seam in two mine fields to the same figure to do comparison and gets Figure 3 (triangle, square and circle represent group D, group E and group F's outburst points of coal seam respectively), and finds that each coal seam outburst has obvious regional. That is $80 \%$ group $\mathrm{D}$, group $\mathrm{E}$ and group $\mathrm{F}$ outburst points are located at north-east wing of Guozhuang anticline, and the outburst happen at the south-west wing are small size. It is showing the controlling compact of geological especially fold to coal and gas outburst.

\section{ANALYSIS FOR COAL AND GAS OUTBURST INFLUENCING FACTOR}

To same extent, the structure controls the storage and distribution of gas. On one hand, structure activity cause the malconformation of gas distribution, which is in favor of the storage and discharging of gas in a certain structure area; on the other hand, the affects of structure stress, complexity of field of stress and the appearing of blocks for different stress concentration in the same structure cause the relative high area and low gas area.

According to the structure characteristics of No.12 mine, we can divide No.12 mine into three gas geological unit, such Niuzhuang syncline, Niuzhuang syncline, Guozhuang anticline wing sharing area, Guozhuang anticline north wing area.

a. Niuzhuang syncline south wing area is belong to monoclinic structure, coal seam buried shallow, which is in favor of the discharge of gas so the gas content is low and pressure is small; when mining, the gas gushing is small and no storage of gas and coal outburst;

b. Guozhuang syncline and Niuzhuang syncline sharing wing area; Niuzhuang syncline is a wide and gentle syncline, inclination angle of coal seam is $5^{\circ} \sim 10^{\circ}$, the stress in syncline axis is squeezing and crack does not grow so gas is easy to gathering. Group F coal seam gas gushing is large near syncline axis; Niuzhuang syncline and Guozhuang anticline sharing wing area are controlled by Niuzhuang syncline, Guozhuang anticline, the original No.11 mine reverse fault, F2 reverse fault and Niuzhuang reverse fault, which structure is complex, gas content is high and gas pressure is large, especially for F six mining area, the relevant gas gushing amount generally is $5 \sim 15 \mathrm{~m}^{3} / \mathrm{t}$. Niuzhuang $-\mathrm{F}_{2}$ reverse fault compressive damage belt and Niuzhuang syncline compressive damage belt coal and gas outburst is extremely high; the 16 times coal and gas outburst in F No.6 mining area happened in compressive damage belt.

$\mathrm{F}_{2}$ reverse fault, the original No.11 mine reverse fault and Niuzhuang reverse fault are compressional fault, the fault surface is closed cut coal seam, which prevent the gas migration to Guozhuang anticline axis and form a good closed structure and is conductive to the preservation of gas (profile map 4, plan 5). Compare F $15-17020$ working face located at the original No.11 mine reverse fault hanging side (in the original No.10 ming syncline axis and fault plan closed structure) and $\mathrm{F}$ 15-17040 working face located at the heading side (in the Guozhuang anticline and fault plan opened structure), the 
result is shown in Figure 6. Through comparison, we see: the gas volume at $F_{15}-17020$ working face is larger than that of $F_{15}-17040$ working face, which is consistent with the control gas storage control of structure.

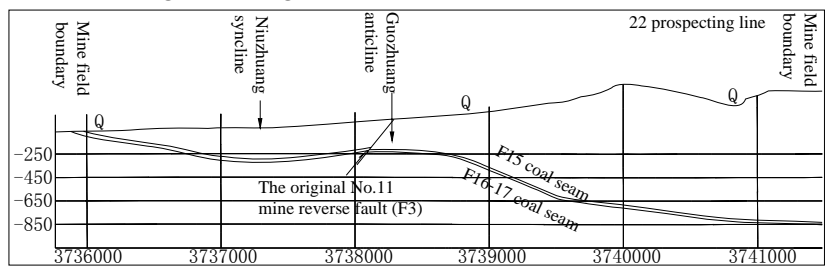

Figure 4. The profile map of 22 prospecting line

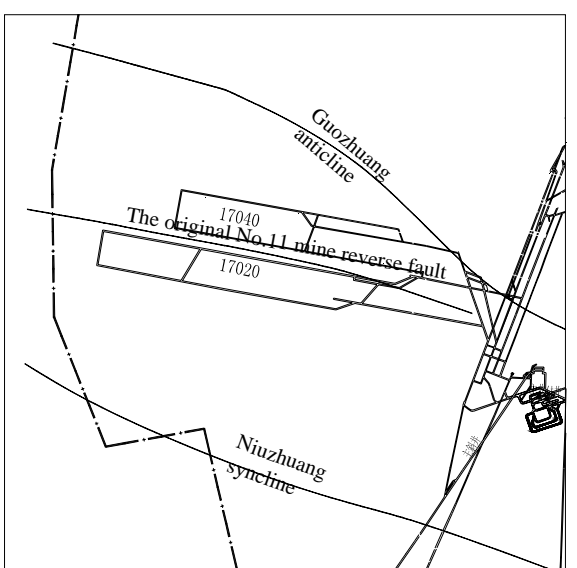

Figure 5. The location diagram of F15-17020 coal face and F15-17040 coal face

TABLE I. THE GAS GUSHING AMOUNT STATISTICS OF F15-17020 WORKING FACE

\begin{tabular}{cccccc}
\hline Date & $2002-4$ & $2002-5$ & $2002-6$ & $2002-7$ & $2002-8$ \\
\hline Absolute gushing amount $\left(\mathrm{m}^{3} / \mathrm{min}\right)$ & 12.95 & 15.83 & 15.74 & 14.76 & 15.40 \\
Relative gushing amount $\left(\mathrm{m}^{3} / \mathrm{t}\right)$ & 3.86 & 8.86 & 7.64 & 6.87 & 6.88 \\
\hline
\end{tabular}

TABLE II. THE GAS GUSHING AMOUNT STATISTICS OF F15-17040 WORKING FACE

\begin{tabular}{cccccccc}
\hline Date & $2003-1$ & $2003-2$ & $2003-3$ & $2003-4$ & $2003-5$ & $2003-6$ & $2003-7$ \\
\hline Absolute gushing amount $\left(\mathrm{m}^{3} / \mathrm{min}\right)$ & 2.91 & 4.32 & 4.23 & 3.78 & 3.97 & 3.51 & 4.38 \\
Relative gushing amount $\left(\mathrm{m}^{3} / \mathrm{t}\right)$ & 2.28 & 3.08 & 2.58 & 4.23 & 3.55 & 3.84 & 4.91 \\
\hline
\end{tabular}

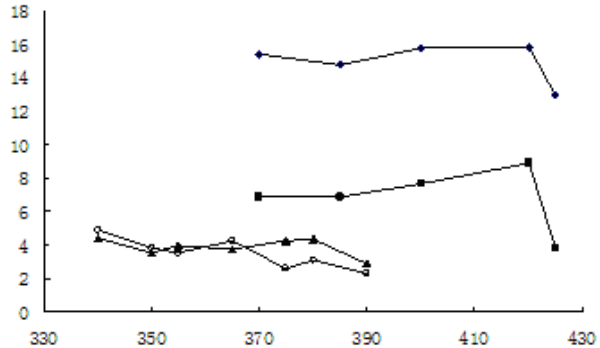

Figure 6. The gas gushing amount of F15-17020 coal face and F15-17040 coal face

The Guozhuang anticline is a fold structure which rise high at the central section of the mine field, which lift up at its south east end and pitches at the north west, running through the entire mine field from the east to the west. Due to the denudation that the anticlinal axis of the Guozhaung anticline suffered, the overlying bedrock becomes thinner and the fracture increases, which is beneficial to the gas dissipation of the Guozhuang anticline axial region; the gas close to the anticlinal axis reduces significantly, and that away from the anticlinal axis increases significantly. As shows in the $F_{15-17-17061}$ working face of the Guozhuang anticline axial region (see Figure 7 ), with the advance of the working face, the gushing amount of the gas that close to the anticlinal axis reduces gradually from $11.30 \mathrm{~m}^{3} / \mathrm{min}$ to $9.20 \mathrm{~m}^{3} / \mathrm{min}$, $7.00 \mathrm{~m}^{3} / \mathrm{min}$ and $2.70 \mathrm{~m}^{3} / \mathrm{min}$ (see figure 8 ) and the regularity is very obvious.

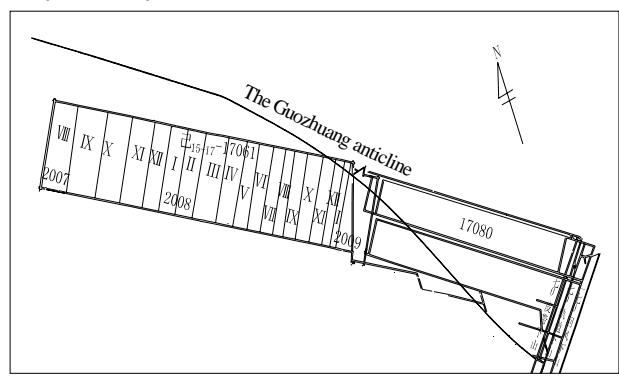

Figure 7. The location plan of F15-17-17061 working face 


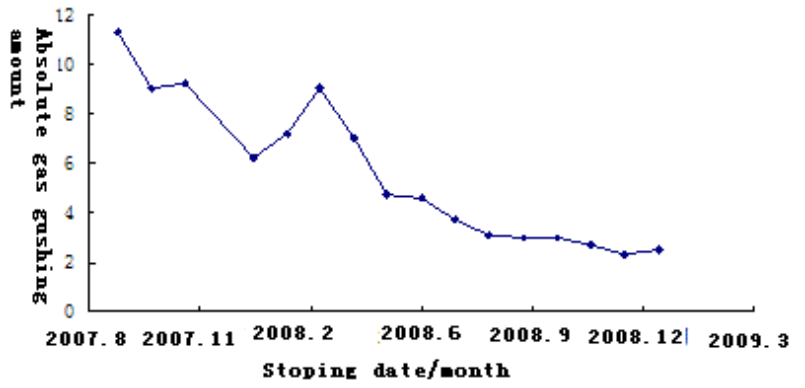

Figure 8. The relationship of gas gushing amount and anticline wheelbase in F15-17-17061 coal face

c. The coal seam occurrence, tectonic coal distribution, gas gushing and the coal and gas outburst in the north wing region of Guozhuang anticline are mainly controlled by Guohzhuang anticline and the Likou syncline. The Guozhuang anticline is a vertical broad fold, and due to its influence, the top and floor coal seam extension fracture within $-300 \mathrm{~m}$ increases. The coal seam has been commonly damaged; the tectonic coal increased and yet the gas has already been released. At the middle depth deeper than $-300 \mathrm{~m}$, due to the control by Guozhuang anticline and Likou syncline, the coal seam depth is very deep; the overlaying bedrock is very thick; and the gas content, gas gushing amount and the danger of the outburst of coal and gas are gradually increasing. Within the scope of coal floor elevation, $-350 \sim-550$, the coal seam inclination of $F_{15}$ is big, and the inclination angle is about $20^{\circ}$. The coal seem is rather thick, the thickness of which is $3.5 \sim 4.5 \mathrm{~m}$. The gas content is great, and gas pressure is huge. The coal and gas outburst is very severe. In this region, there have been 9 outbursts of coal and gas.

\section{CONCLUSION}

1. In No. 12 mine, nearly all the coal and gas outbursts are closely related to the structure, which shows a favorable divisional and zonal regularity.

2. Due to the control by the regions such as Niuzhuang syncline and Guozhuang anticline as well as the Likou anticline, the outburst of coal and gas in the well field of
No.12 mine has a high zonality. The coal and gas outbursts of group $\mathrm{F}$ are distributed in 3 regions: the coal and gas outburst zone in the F6 mining area; the coal and gas outburst zone of the coal floor elevation $-350 \mathrm{~m} \sim$ $-600 \mathrm{~m}$ and the three level deep coal and gas outburst zone.

3. The coal and gas outburst zone in the F6 mining area is controlled by the geological structure such as the Niuzhuang syncline, the Guozhuang anticline, the Niuzhuang reverse fault, F2 reverse fault and the original No. 11 mine reverse fault. The structure is very complex, which is beneficial to the gas occurrence. The gas content is profound; the tectonic coal increases significantly and the stress concentrates greatly. The coal and gas burst has a concentrated distribution at the pinching-out area.

\section{REFERENCES}

[1] ZHANG Zi-min.WU Yin. Tectonic-level-control rule and area-dividing of coalmine gas occurrence in China [J]. Earth science forntiers,2013,(02):237-245.

[2] YU Bu-fan. Discussion of coal and gas outburst mechanism[J].Coal Science and Technology, 1979(8): 34-42.

[3] X u F e n g y i n.The Quantitative Research Onthe Palaeotectonic Atressfiela And Its Contr To Coal And Gas Outburst,Scientia Geologica Sinica. [J]. Scientia Geologica Sinica, 1995,30(1): 71-84.

[4] YU Bu-fan.Mine gas hazards prevention and use technical manual[M].Beijing: Coal Industry Press, 2000.

[5] HANJun, ZHANGHong-wei ,Discussion of coal and gas outburst mechanism of syncline[J].Journal of China Coal Society, 2008, 33(8): 908-913.

[6] LI Tao, ZHANG Hong-wei. Controlling effect of tectonic stress field on coal and gas outburst[J]. JOURNAL OF XI'AN UNIVERSITY OF SCIENCE AND TECHNOLOGY, 2011,31(6):715-718.

[7] LI Tao, ZHANG Hong-wei. Controlling effect of tectonic stress field on coal and gas outburst[J]. JOURNAL OF XI'AN UNIVERSITY OF SCIENCE AND TECHNOLOGY, 2011,31(6):715-718.

[8] ZHANG Zimin, ZHANG Yugui. Gas geology [M]. Xuzhou: China University of Mining and Technology Press,2009.

[9] CHENG Yuan-ping, ZHANG Xiao-lei, WANG Liang. Controlling effect of ground stress on gas pressure and outburst disaster[J]. Journal of Mining \& Safety Engineering,2013,3(3):408-414. 